\title{
Breast Cancer in Older Patients
}

\author{
Chair: $\quad$ Raimund Jakesz, Wien \\ Participants: Christian Jackisch, Offenbach Walter Jonat / Nikolai Maass, Kiel \\ Peter Schrenk, Linz Rainer Souchon, Hagen Michael Stierer, Wien \\ Susanne Taucher, Innsbruck
}

Breast cancer patients older than 70 years are usually not accrued to clinical trials. There are only few exceptions - including ABCSG 8 - which accrued patients up to 80 years. The mean age of patients developing breast cancer in Western countries is increasing and therefore a standardized procedure for so-called 'older patients' is urgently required.

\section{Question 1: Do you regard screening mammography for women older than $\mathbf{7 0}$ years of age as indicated?}

Jackisch: As long as data on mortality reduction are missing I vote no.

Jonat/Maass: In the age-group of women older than 70 years we follow an individualized screening strategy. Depending on preference and morbidity of the patient mammography screening is optional. Several national screening programs include patients up to the age of 75 . Both sensitivity and specificity of mammographies are higher in this age group because of the low density of breast parenchyma and the higher incidence of breast cancer in this age groups.

Schrenk: Yes. Some trials show that women $>70$ benefit from screening. However, routine screening in these patients depends on general health, co-morbidity, and life expectancy.

Souchon: The average life expectancy in Germany for women over 70 years of age is 16 years, for women over 75 it is 12 years, and for 80 -year-old women it is 8.5 years. Although there are no trials as to improvement of survival in elderly women through mammography screening, this should be offered - not least on ethical reasons, having in mind the fact that more than half of breast cancers are discovered in women older than 65 years. This type of screening can be compared to other preventive medical checkups which are not age-restricted, like screening for prostatic or colocrectal cancer. Exposure to radiation in elderly patients is certainly irrelevant, more important would be a restriction to patients whose life expectancy is not reduced by other (chronic) diseases.

Stierer: Experience that may result in evidence-based information is rather limited in terms of mammography screening given to women beyond the age of 70. In The Netherlands, for example, the upper age limit for the national screening program was extended to 75 years between 1989 and 2000; and since 2002, breast cancer mortality has been dropping in this age group. Similar outcomes have been seen in Sweden. In view of the age-dependent increase in incidence and prolonged life expectancy - healthy 75-year-old women may well live another 12 years on average - it appears reasonable to extend the age limit for mammography screening.

Taucher: Unfortunately we have no evidence for any benefit of screening mammography in women older than 70 years. I would recommend regular clinical examination of the breast as well as mammography and ultrasound in elderly women. We may achieve even better results with this approach than by screening.

\section{Question 2: Do you regard malignant tumors in patients who are $\mathbf{7 0}$ years and older as biologically less aggressive?}

Jackisch: Not at all - this interpretation might lead to undertreatment in this age group. Unfortunately most of the clinical trials excluded patients of that age group from participation, regardless of the fact that most breast cancers occur in elderly women.

\begin{tabular}{ll}
\hline KARGER & ๑ 2007 S. Karger GmbH, Freiburg \\
Fax +49 7614520714 & Accessible online at: \\
$\begin{array}{l}\text { E-mail Information@Karger.de } \\
\text { www.karger.com }\end{array}$ & www.karger.com/brc
\end{tabular}


Jonat/Maass: Patients 70 years of age on average have a life expectancy of 15-18 years. In otherwise healthy patients without severe co-morbidities we often suggest the same treatment options as in younger individuals. We do not distinguish patients with biologically less aggressive tumors.

Schrenk: Yes, more tumor patients in this age group present with favorable grading, receptor positivity, and negative Her2-neu status.

Souchon: There are no conclusive data which would support this assumption in postmenopausal women. More important are the well-known facts like tumor stage at the time of diagnosis and biological subtype.

Stierer: Due to the acknowledged higher percentage of tumors with good prognostic features $(80 \%$ hormone receptor positivity, HER2/neu negativity, low Ki67, rare lymph node involvement), breast cancers emerging beyond the age of 70 by nature are biologically less aggressive.

Taucher: Malignant tumors in the elderly do not behave less aggressively. The issue whether or not a malignant tumor grows fast depends on a variety of prognostic markers in breast cancer. There is a correlation between age and some prognostic features in very young patients, but not in the elderly.

\section{Question 3: Is mastectomy the preferred choice of surgical treatment in patients of this age group?}

Jackisch: Not at all - We have learned from the Swiss group [Bouchardy et al., 2003] that breast conservation in the elderly is the best option. Without any doubt we are not referring to the fact that in this population a very complicated surgical approach is requested. In most of the cases a segmentectomy might be recommended. In case of surgery, proper radiation therapy should be discussed with and offered to the patients.

Jonat/Maass: Patients in this group will be offered the same surgical procedures as younger patients, in this case, if possible breast conserving surgery. The relation of breast conserving surgery in patients older than seventy is almost the same as in younger patients. Still the patient is offered a choice, especially if they decline postoperative breast irradiation.

Schrenk: No, surgical treatment should be based on same criteria as in younger patients. As seen from our patients the rate of patients wishing to have their breast preserved is as high as in younger women (even with oncoplastic surgery and immediate reconstruction - we see no contraindication for a reconstruction with respect to age, but comorbidity). In few cases I would also spare the patients a radiation.
Souchon: If there is a possible curative therapeutic intention, mastectomy should not be first choice. The informed patient's wish decides, meaning that if there is a possibility of breast conserving surgery, the patient must be informed, willing and medically fit for a post-op radiotherapy (RT) of several weeks.

Stierer: Personally, I do not believe we have a definitive answer to that as yet. On the patients' side, we encounter both the women's categorical wish to undergo breast-conserving treatment, including breast reconstruction following ablation, as well as unemotional management in terms of ablative procedures, particularly in connection with the possibility of avoiding postoperative radiotherapy. Considering the fact that diagnosis is quite frequently delayed in older women, we are left with limited possibilities as to preoperative chemotherapy - and are thus basically restricted to mastectomy. In hormone receptor-positive cases, hormonal therapy may be considered. In principle, however, breast-conserving procedures should also be offered as first option in this age group whenever feasible.

Taucher: The choice of surgical treatment should be based on individual factors. Age is definitely just one of the factors we have to take into consideration. The cut-off at 70 years may fit for some women, but not for all of them. If breast conservation is possible in a patient older than 70 years, comorbidity, performance status, availability of radiation therapy and the patient's choice after careful counseling should influence our surgical treatment.

\section{Question 4: Is there an indication for postoperative treatment in patients $>70$ with ER+ PgR+, DCIS, treated with breast-conserving surgery?}

Jackisch: I have to guess in the absence of data from randomized trials; for this issue we must await the data from the ICE trial and the ongoing CASA trial. My preference in endocrine responsive DCIS would - even in the elderly - be tamoxifen $20 \mathrm{mg}$ a day for 5 years. I would caution my colleagues to reflect on the administration of 3rd generation aromatase inhibitors in this setting.

Jonat/Maass: There is no difference in this subgroup compared to younger patients concerning the indication for radiotherapy. Following the newest guidelines, nearly all DCIS treated with breast conserving surgery will receive radiotherapy. Systemic treatment with tamoxifen will be offered very conservatively in this group. Prevention strategies, like in the IBIS II study are not indicated in the subgroup.

Schrenk: Yes, there is an indication for postoperative treatment, but only for radiation treatment in patients with DCIS III and tiny margins. Otherwise good quality surgery with 
wide margins of $10 \mathrm{~mm}$ or more in DCIS is enough treatment in these patients. I would not recommend endocrine treatment in these DCIS patients.

Souchon: Regarding the subgroup of patients with DCIS who underwent breast-conserving surgery, post-op RT is much more important concerning local tumor control than tamoxifen therapy. There are two specific studies with divergent results, namely the UKCCR DCIS trial ( $\mathrm{n}=1701$ pats; [Houghton et al., 2003]) and the NSABP B24 trial ( $\mathrm{n}=1804$; [Fisher et al., 1999, 2001]). In the UKCCR DCIS trial no evidence of an advantage of tamoxifen therapy was seen.

Stierer: All studies have shown that there is no subgroup in DCIS that does not profit from postoperative irradiation in terms of local relapse prevention. However, radiotherapy may potentially be omitted in this age group in the presence of G1 and $\mathrm{G} 2$ lesions $(<2.5 \mathrm{~cm}$ in diameter), clear surgical margins $>5 \mathrm{~mm}$, and annual mammography checkups following patients' informed consent. There seems to be no generally imperative indication for tamoxifen in this setting. This treatment option is only considered in the presence of extensive G3 tumors treated with breast-conserving surgery, including individual risk assessment (cormorbidity, status post extirpation of the uterus).

Taucher: The indication for postoperative treatment in this patient cohort is determined by size of DCIS and resection margin. Application of the Van Nuys Prognostic Index is useful in all patient subgroups. Radiation as well as endocrine treatment should be offered to these patients.

\section{Question 5: Is there an age limit for postoperative irradiation after breast conserving surgery?}

Jackisch: As long as we have no data we have to generate the missing evidence. Even surgery is an option for local control of the disease. Thus, surgeons should always consider surgical procedures along with the proper radiotherapy in operable breast cancer, regardless of the age of the patient [Bouchardy et al., 2003].

Jonat/Maass: All patients, independent of age, receive postoperative irradiation after breast conserving surgery. New radiation strategies in the field of brachytherapy techniques, such as the 'Mammosite' catheter or 'interstitial' approaches are under investigation in studies.

Schrenk: No, there is no age limit for patients with invasive carcinoma. However, some patients may be spared radio therapy (e.g., patients with decreased life expectancy, high co-morbidity, favorable histology (tubular, papillary), G1 tumor, tumor $<1 \mathrm{~cm})$.
Souchon: As compared to tamoxifen alone, whole breast irradiation (WBR) significantly reduced the risk of breast cancer recurrence after breast conserving therapy in women $>70$ years of age with ER-positive breast cancers [Hughes et al., 2004]. According to the recent meta-analysis of the EBCTCG 2005 [Clarke et al., 2005] the proportional reduction of local recurrence by whole breast irradiation is $70 \%$ for each age group. Also, the significant impact of a higher radiation dose is noteworthy. Addition of a boost irradiation restricted to the tumor bed to whole breast irradiation is beneficial in all age groups. The 7-year recurrence rates are reduced by half, independently of patient's age, which has been demonstrated recently [Antonini et al., 2007; Bartelink et al., 2007]. Still, it has been proven that radio therapy effected absolute reduction of risk of local recurrence is nearly doubled in patients younger than 50 years of age in comparison with older women. Nevertheless, restricted to women who have ER-positive early breast cancer, patients as well as physicians have to weigh the increased risk of local relapse against the inconvenience of radio therapy, and even more, additional boost irradiation.

Stierer: Stringent data have shown that postoperative radiotherapy can be omitted, without compromising secondary mastectomy rates or overall survival, in $\mathrm{R} 0$ resected $\mathrm{T} 1$ tumors, negative lymph node status, positive hormone receptor status, preferably G1 and G2 grading, and with antihormonal treatment. On the other hand, I believe establishing an upper age limit for postoperative irradiation should always be done on an individual basis.

Taucher: The benefit of postoperative radiation is mainly local control. The life expectancy of a 70-year-old woman may be 20 years. Hence, even older patients benefit from postoperative radiation after breast conserving therapy.

\section{Question 6: Is age a limiting factor for postoperative chemotherapy?}

Jackisch: This is the most important issue - as most of the elderly patients are suffering from endocrine responsive tumors chemotherapy alone is a rare issue. I personally prefer the chemo-endocrine sequence, most preferable we use the ICE protocol. In general, and specifically in that age group, physicians should explore the overall burden of disease and quality of life along with preexsisting co-morbidities that might jeopardize the delivery of chemotherapy.

Jonat/Maass: In general, age is not a limiting factor if patients have no co-morbidities. Especially lymph-node positive and hormone-receptor negative patients will receive chemotherapy (almost) independent of age. The indication of chemotherapy has to be discussed with the patient based on possible co- 
morbidities. Balance between benefit and side effects is important. Tools like 'adjuvant-online' might be helpful to discuss the potential benefits of chemotherapy.

Schrenk: No, age does not limit the use of post-operative chemotherapy where it is indicated. It is not indicated when life expectancy is decreased or in patients with co-morbidities.

Souchon: This has to be decided individually, taking into account comorbidity and depending on the intention of treatment. While adjuvant treatment demands a geriatric assessment to ensure eligibility of the patient, this question does not pertain to palliative treatment.

Stierer: Administration of cytotoxic chemotherapy in addition to antihormonal treatment yields marginal improvements in hormone receptor positive patients above the age of 70 $(0.4-0.7 \%$ mortality reduction with tumor-free lymph nodes and $0.7-1.2 \%$ reduction with $1-3$ involved nodes) while it clearly increases toxicity. The indication is certainly individualized in patients with more than 3 involved nodes, or 1-3 involved nodes and HER2/neu overexpression; life expectancylimiting comorbidity should also be given attention. Such treatment is equally effective among women above 70 with hormone receptor negative tumors and those aged 60-69 years, but it is associated with clearly increased cardiac and bone marrow toxicities. The indication is individualized in lymph node-negative high-risk patients (tumor size $>3 \mathrm{~cm}$, G3) and in the presence of lymph node positive tumors. Administration of established cytotoxic agents beyond the age of 80 seems highly questionable in current adjuvant treatment.

Taucher: Side effects of postoperative chemotherapy are often severe in the elderly. We have to take into consideration that older women often suffer from a variety of other diseases. The higher incidence of heart diseases in this patient subgroup is a distinct limitation to postoperative chemotherapy in this agegroup. Although we have some chemotherapeutical agents which may be suitable in patients suffering from a heart disease, age is an additional issue. Postoperative treatment and particularly postoperative chemotherapy must be tailored for the individual patient.

\section{Question 7: Do you regularly follow patients over 70 years of age postoperatively after breast cancer surgery?}

Jackisch: Yes we do, as the German guidelines demand a 5-year-follow-up of breast cancer patients, focusing on clinical assessment and mammography. The interval in the first 2 years is every 3 months. In year 3-4 a reassessment is requested every 6 months, thereafter the checkup procedures are prescheduled every 6 months.
Jonat/Maass: This age group is included in the follow-up program like all other patients with breast cancer. We invite women to follow-up investigations in yearly intervals. In patients with higher risk also in 6-monthly intervals. Only this approach ensures long-term quality of results.

Schrenk: Yes, as with younger patients we do a regular followup and as in younger patients some attend regularly and others never after surgery.

Souchon: In Germany, it is the obligation of the radiation oncologist to do follow-up examinations of every patient who has been subjected to radiotherapy. This is rooted in a law regulating radiation protection and cannot be substituted by doctors other than radiation oncologists.

Stierer: Patients older than 70 years should be given life-long postoperative follow-up, with the womens' general condition being the only limiting factor.

Taucher: We perform a regular follow-up in all our patients. Some of my best patients are considerably older than 70 years. I can't imagine any reason to stop regular controls at a distinct age.

\section{References}

Antonini N, Jones H, Horiot JC, et al.: Effect of age and radiation dose on local control after breast conserving treatment: EORTC trial 22881-10882. Radiother Oncol 2007;82:265-71.

Bartelink H, Horiot JC, Poortmans PM, et al.: Impact of a higher radiation dose on local control and survival in breast-conserving therapy of early breast cancer: 10year results of the randomized boost versus no boost EORTC 22881-10882 trial. J Clin Oncol 2007;25:3259-65.

Bouchardy C, Rapiti E, Fioretta G, et al.: Undertreatment strongly decreases prognosis of breast cancer in elderly women. J Clin Oncol 2003;21:3580-7.

Clarke M et al. for the Early Breast Cancer Trialists' Collaborative Group: Effects of radiotherapy and of differences in the extent of surgery for early breast cancer on local recurrence and 15-year survival: an overview of the randomised trials. Lancet 2005;366:2087-2106.

Clarke M: Meta-analyses of adjuvant therapies for women with early breast cancer: the Early Breast Cancer Trialists' Collaborative Group overview. Ann Oncol 2006; Suppl 10:x59-x62.

Early Breast Cancer Trialists' Collaborative Group (EBCTCG): Effects of chemotherapy and hormonal therapy for early breast cancer on recurrence and 15-year survival: an overview of the randomised trials. Lancet 2005;365:1687-717.

Fisher ER, Dignam J, Tan-Chiu E, Costantino J, Fisher B, Paik S, et al.: Pathologic findings from the National Surgical Adjuvant Breast Project (NSABP) eight-year update of protocol B-17: intraductal carcinoma. Cancer 1999;86:429-38.

Fisher B, Land S, Mamounas E, Dignam J, Fisher ER, Wolmark N: Prevention of invasive breast cancer in women with ductal carcinoma in situ: an update of the national surgical adjuvant breast and bowel project experience. Semin Oncol 2001;28:400-418

Houghton J, George WD, Cuzick J, Duggan C, Fentiman IS, Spittle M for UK Coordinating Committee on Cancer Research (UKCCCR) Ductal Carcinoma in situ (DCIS) Working Party on behalf of DCIS trialists in the UK, Australia, and New Zealand: Radiotherapy and tamoxifen in women with completely excised ductal carcinoma in situ of the breast in the UK, Australia, and New Zealand: randomised controlled trial. Lancet 2003;362:95-102.

Hughes KS, et al. Cancer and Leukemia Group B; Radiation Therapy Oncology Group; Eastern Cooperative Oncology Group: Lumpectomy plus tamoxifen with or without irradiation in women 70 years of age or older with early breast cancer. N Engl J Med 2004;351:971-7. 
Jahrbuch Statistisches Bundesamt für die Bundesrepublik Deutschland. www.desta tis.de

Mandelblatt J: To screen or not to screen older women for breast cancer: a new twis on an old question or will we ever invest in getting the answers? J Clin Oncol 2007;25:20991-2.

\section{Participants}

Prof. Dr. med. Christian Jackisch

Klinikum Offenbach

Frauenklinik

Starkenburgring 66

63069 Offenbach, Germany

E-mail christian.jackisch@klinikum-offenbach.de

Prof. Dr. med. Walter Jonat

Prof. Dr. med. Nicolai Maass

Klinik für Gynäkologie und Geburtshilfe

Universitätsklinikum Schleswig-Holstein, Campus Kiel

Michaelisstraße 16

24105 Kiel, Germany

E-mail jonat@email.uni-kiel.de /nmaass@email.uni-kiel.de

Prof. Dr. med. Rainer Souchon

Klinik für Strahlentherapie und Radioonkologie

Allgemeines Krankenhaus Hagen gGmbH

Grünstr. 35

58095 Hagen, Germany

E-mail souchon@akh-hagen.de

Doz. Dr. med. Peter Schrenk

2. Chirurgische Abteilung

Allgemeines Krankenhaus Linz

Krankenhausstrasse 9

4020 Linz, Austria

E-mail peter.schrenk@liwest.at

Prof. Dr. med. Michael Stierer

Chirurgische Abteilung

Hanusch Krankenhaus

Heinrich Collin Strasse 30

1140 Wien, Austria

E-mail michael.stierer@wgkk.sozvers.at

Univ. Prof. Dr. med. Susanne Taucher

Universitätsklinik für Frauenheilkunde

Anichstrasse 35

6020 Innsbruck, Austria

E-mail susanne.taucher@i-med.ac.at 